\section{Injectable local anaesthesia for IUD/IUS fittings}

At a recent Margaret Pyke update, among more than 200 general practitioners and family planning clinic doctors, it was apparent that less than $5 \%$ use injectable local anaesthesia (ILA) for their intrauterine device/intrauterine system (IUD/IUS) fittings. As we now profess to be patient centred, this is difficult to comprehend.

There are many reasons doctors choose not to use ILA nor even, against Faculty guidance, to offer it. These range from "no time", "the pain only lasts a short time" to "I am so good I don't cause pain". Some believe the injections to be more painful than the insertion and even the attitude that women are hysterical still persists. This is all nonsense.

I would like to propose that the use of injectable local anaesthesia should be the default position for IUD/IUS fittings from which women can, if they wish, opt out. Why?

1. More than $50 \%$ - the majority of women - experience some pain during IUD/IUS insertion. For many this is "the worst pain I have ever experienced". Fundal cramping cannot be prevented by ILA but the cervical component is the more extreme and overwhelming pain and is unnecessary and avoidable. Parity can mitigate this but by no means always.

2. ILA is simple, safe, quick and painless to administer and immediately effective. The largely ineffective alternative, lignocaine gel, takes $5-10$ minutes to give any effect at all and ironically has greater potential to cause pain by dilation of the cervix during its application through the quill.

3. As for time consuming, it is easy to argue that without overwhelming cervical pain there will be less cervical shock. Thus surgeries are less likely to be clogged by women collapsing, experiencing unpleasant and avoidable vasovagal attacks.

4. Against a background of attempting to promote intrauterine contraception as a long-acting reversible contraceptive we do not want women to have a painful experience. We want women to tell their friends - for word of mouth is how the word will be spread - that the insertion was painless. Only then can we look forward to increasing numbers of women choosing intrauterine contraception.

5. Let us not forget that if men were to have a device inserted through their genitalia they would demand general anaesthetic as a hospital inpatient. Who is hysterical?

There was a time when ILA was not the default position in dentistry.
Thankfully it now is. No dentist asks whether we would like ILA, they simply give it. I look forward to the enlightened time when we in sexual health care do the same. Enough women have suffered unnecessarily. Women can opt out of the use of ILA though most are reassured that the injections into the cervix - a frightening prospect - are less uncomfortable than those in dentistry.

Auditing my last 140 insertions, of which $80 \%$ are nulliparous, I used ILA in 135 women, over $96 \%$. I used gel in two women and no LA in three. Of the 135 women in whom I used ILA, five had some form of mild to moderate vasovagal experience. Thus over $96 \%$ had a relatively easy insertion.

Lignocaine is a wonderful thing. Why ignore it and its great advantages?

Sam Hutt, MA MB, FFSRH

Margaret Pyke Centre, London, UK; hankwangford@gmail.com

Competing interests None.

J Fam Plann Reprod Health Care 2011;37:59. doi:10.1136/fprhc.2010.0037 